\title{
Biosensing with backscattering interferometry
}

Henrik S. Sørensen, Niels B. Larsen, Peter R. Hansen, Peter E. Andersen, and Darryl J. Bornhop

Monitoring biochemical interactions can serve as the basis for many diagnostic techniques.

Backscattering interferometry (BSI) ${ }^{1}$ uses light interaction with a microfluidic channel (see Figure 1) to measure temporal changes in refractive index (RI), which can be caused by the channel's bulk material properties or solutes (dissolved substances). It enables high-resolution investigation of unlabeled proteins, both in solution ${ }^{2}$ and adsorbed (or immobilized) to a surface. ${ }^{3}$ Upon coherent-laser illumination of a microfluidic channel, a highly modulated fringe pattern is produced perpendicular to the channel. Its bright and dark features shift position with changes in the RI of the sampled liquid, and monitoring this shift forms the basis of BSI.

Using BSI in fused-silica capillary tubes enables detection ${ }^{4}$ of RI changes of order $10^{-9}$ (see Figure 2). Modeling the capillarytube optical train was found useful for performing absolute RI measurements with high accuracy. ${ }^{5,6}$ One major step forward in BSI development was the transition from capillary tubes to microfluidic networks. The microfluidic channels can be fabricated in a number of ways including standard SU-8 (a viscous polymer) photoresist procedures. By casting and curing polydimethylsiloxane (PDMS), these microstructures can be replicated. In the PDMS flow channel, immobilization prepares the chip for biochemical-interaction experiments.

Photobiotin forms a covalent bond to the oxidized PDMS surface. And, ExtrAvidin, which reacts with biotin, provides a useful surface for heterogeneous binding studies. Binding biotinylated protein A and DNA allows preparation of a surface for BSI analysis. The immobilized molecule targets will now interact with solute samples as they travel through the channels. Two of the systems we studied were protein-A binding to the Fc fragment (the crystallizable region of an antibody that interacts with cell-surface receptors) of human immunoglobulin G (IgG) and a 30-mer DNA strand for hybridization experiments. In addition, we performed BSI hybridization studies on the

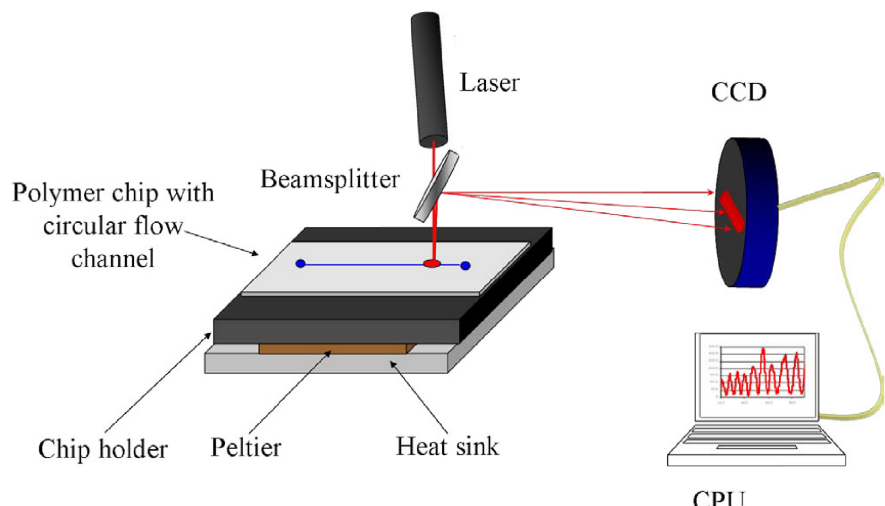

Figure 1. Backscattering interferometry setup. CPU: Central processing unit.

complementary strand, both with and without fluorescein isothiocyanate label. The high sensitivity of the BSI sensor enabled us to detect a change in binding affinity between labeled and non-labeled DNA strands.

Monitoring and determining the rate and affinity of biomolecular interactions, such as antigen-antibody binding events, can serve as the basis for many diagnostic techniques and aid in development of novel therapeutics. We recently found that BSI can detect label-free and free-solution binding with unprecedented limits in microfluidic devices. Featuring a unique optical train, BSI allows near real-time determination of binding constants spanning six decades from micro- to picomoles. Such a wide dynamic range is relevant because most circulating molecules of pathophysiological interest in human disease are found at levels from femto- to micromoles. We quantified equilibrium dissociation constants for protein A and IgG, interleukin-2 with its monoclonal antibody, calmodulin with $\mathrm{Ca}^{2+}$ (a small molecule inhibitor), the protein calcineurin, and the M13 peptide. The high sensitivity of BSI and small volumes of microfluidics allowed us to perform the entire calmodulin experiment with 200 pM of solute. 


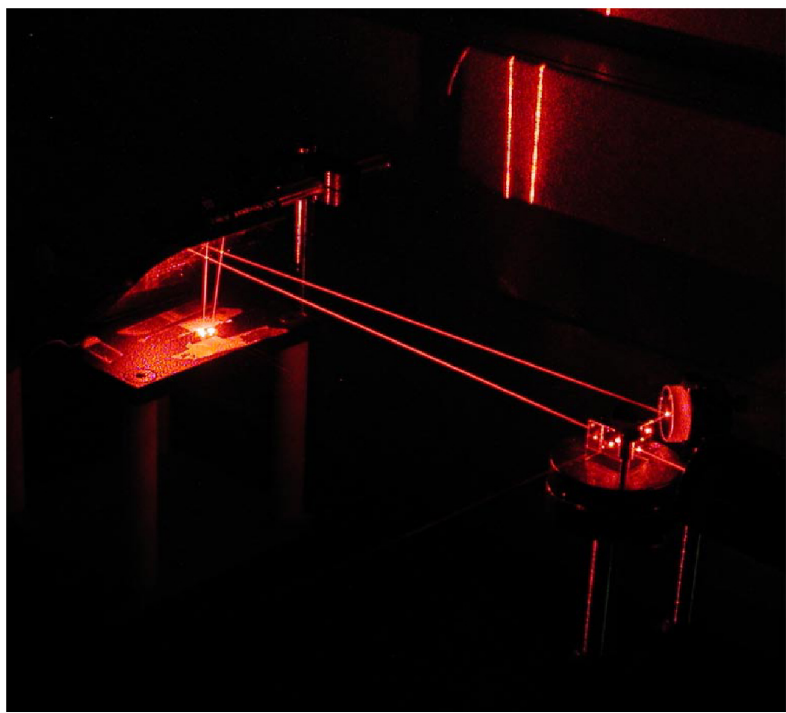

Figure 2. Dual-capillary setup, showing two laser beams illuminating two capillaries.

In summary, BSI has the highest figure of merit among candidate protein-detection schemes, given the combination of sample volume, time, sensitivity, cost, and system simplicity for label-free protein-protein interaction studies in solution. Its capacity to measure essentially any circulating disease marker or mediator in blood samples could lead to the development of numerous clinical applications. The technique may also be applied to other fields for analysis of small volumes of liquid samples, including drug discovery and environmental monitoring. We will next investigate the feasibility of using BSI as a bedside diagnostic tool.

\section{Author Information}

\section{Henrik S. Sørensen and Peter E. Andersen \\ Department of Photonics Engineering \\ Technical University of Denmark \\ Roskilde, Denmark}

Henrik Sørensen holds a postdoctoral position. His main research area is optical biosensing based on BSI, including optical simulations and applications to detect biomolecular interactions in solutions without labeling the molecules.

Peter Andersen is a research professor and also holds an appointment as visiting professor at Lund University (Sweden). His primary research interest is biophotonics, particularly optical coherence tomography and BSI for biosensing applications.

\author{
Niels B. Larsen \\ Department of Micro- and Nanotechnology \\ Technical University of Denmark \\ Roskilde, Denmark
}

Niels Larsen is a research professor. His research area is chemical and topographical surface engineering of polymer materials, with a strong background in advanced surface analysis.

\section{Peter R. Hansen \\ Department of Cardiology \\ Gentofte Hospital \\ Hellerup, Denmark}

Peter Hansen is a consultant in invasive cardiology. He is also medical advisor for the Danish Medicines Agency. His primary research interests are reperfusion injury, coronary microcirculation, inflammatory mechanisms in cardiovascular diseases, atherogenesis, and invasive cardiology.

\section{Darryl J. Bornhop \\ Department of Chemistry \\ Vanderbilt University \\ Nashville, TN}

Darryl Bornhop is a professor. His main research interests are nanoscale chemical and biochemical sensing and molecular imaging through multidisciplinary bioanalytical and biomedical research. He invented BSI.

\section{References}

1. D. J. Bornhop, Microvolume index of refraction determinations by interferometric backscatter, Appl. Opt. 34 (18), pp. 3234-3239, 1995.

2. D. J. Bornhop, J. C. Latham, A. Kussrow, D. A. Markov, R. D. Jones, and H. S. Sørensen, Free-solution, label-free molecular interactions studied by back-scattering interferometry, Science 317, pp. 1732-1736, 2007.

3. J. C. Latham, D. A. Markov, H. S. Sørensen, and D. J. Bornhop, Photobiotin surface chemistry improves label-free interferometric sensing of biochemical interactions, Angew. Chem. Int'l Ed. 45, pp. 955-958, 2006.

4. Z. Wang and D. J. Bornhop, Dual-capillary backscatter interferometry for highsensitivity nanoliter-volume refractive index detection with density gradient compensation, Anal. Chem. 77 (24), pp. 7872-7877, 2005.

5. H. S. Sørensen, H. Pranov, N. B. Larsen, D. J. Bornhop, and P. E. Andersen, Absolute refractive index determination by microinterferometric backscatter detection, Anal. Chem. 75, pp. 1946-1953, 2003.

6. H. S. Sørensen, N. B. Larsen, J. C. Latham, D. J. Bornhop, and P. E. Andersen, Highly sensitive biosensing based on interference from light scattering in capillary tubes, Appl. Phys. Lett. 89, p. 151108, 2006. 